Предавање ${ }^{1}$

061.1EU:34.13

doi:10.5937/zrpfns46-3118

O. Univ. Prof. DDr. Heinz Mayer

Institut für Staats- und Verwaltungsrecht der Universität Wien

\title{
Der Vorrang des Unionsrechts und die Rechtskraft nationaler Individualrechtsakte ${ }^{2}$
}

Der moderne Rechtsstaat ist vom Dualismus von genereller und individueller Norm bestimmt: Gerichte und Verwaltungsbehörden haben die vom Gesetzgeber geschaffenen generellen Normen zu individualisieren und zu konkretisieren. Am Ende dieses Prozesses steht eine verwaltungsbehördliche Entscheidung - in Österreich als Bescheid bezeichnet - oder ein gerichtliches Urteil. Im Regelfall unterliegen diese Rechtsakte einer Überprüfung durch mehrere Instanzen. Nach Beendigung des verwaltungsbehördlichen oder gerichtlichen Instanzenzuges sind diese Entscheidungen im Allgemeinen unanfechtbar und unabänderbar.

Die Überprüfbarkeit verwaltungsbehördlicher oder gerichtlicher Rechtsakte im Instanzenzug soll deren Richtigkeit - will heißen: Gesetzmäßigkeit sicherstellen. Hat die letzte Instanz entschieden, soll Rechtssicherheit eintreten: Die Betroffenen sollen sich auf den Bestand dieser Rechtsakte verlassen können. Dies gilt auch dann, wenn der letztinstanzliche Rechtsakt möglicherweise nicht der Rechtsordnung entspricht und daher rechtswidrig ist. Das positive Recht versucht zwar die Rechtmäßigkeit der Vollziehung durch ein Rechtsschutzsystem sicherzustellen, nimmt aber im Interesse der Rechtssicherheit letztlich bestimmte Rechtswidrigkeiten in Kauf. Die Rechtskraft - gelegentlich auch als „Bestandskraft“ bezeichnet - kann im Allgemeinen nur mehr bei sehr schweren Mängeln durchbrochen werden; etwa dann, wenn der betreffende Akt

\footnotetext{
${ }^{1}$ Проф. Хајнц Мајер је 24. октобра 2012. године на Правном факултету у Новом Саду одржао предавање на бази објављеног текста.

${ }^{2}$ Dieser Text wird mit freundlicher Genehmigung des Manz-Verlages Wien publiziert; er erscheint auch in der Schriftenreihe der Forschungsstelle für Europäische Privatrechtsentwicklung der Rechtswissenschaftlichen Fakultät der Universität Wien.
} 
durch strafbare Handlungen herbeigeführt wurde oder in seinen Auswirkungen geradezu unerträglich wäre ${ }^{3}$ ).

Rechtsrichtigkeit und Rechtskraft sind auch Prinzipien des Unionsrechts. Auch das Unionsrecht ist von diesen Prinzipien beherrscht und nimmt in Kauf, dass die Rechtskraft in bestimmten Fällen der Rechtsrichtigkeit vorgeht.

Spezifische Überlegungen werden im Zusammenspiel zwischen dem Unionsrecht und dem nationalen Recht erforderlich. Die Vollziehung des Unionsrechts fällt im Wesentlichen in die Zuständigkeit nationaler Behörden; lediglich in Ausnahmsfällen erfolgt der Vollzug des Unionsrechts durch Organe der Union.

Beim Vollzug des Unionsrechts durch nationale Behörden gewinnen zwei Prinzipien besondere Relevanz: Auf der einen Seite betont der EuGH eine strikte Vorrangwirkung des Unionsrechts vor nationalem Recht jedweder Stufe. In der Entscheidung Costa/E.N.E.L. betont der EuGH bereits im Jahr 1964, dass dem Unionsrecht ,keine wie immer gearteten innerstaatlichen Rechtsvorschriften vorgehen können ..." $\left.{ }^{4}\right)$. Alle nationalen Organe - Gerichte ebenso wie Verwaltungsbehörden - haben unionswidriges nationales Recht unangewendet zu lassen. Auf der anderen Seite betont der EuGH den Grundsatz der Verfahrensautonomie der Mitgliedstaaten. In ständiger Rechtsprechung betont der EuGH, dass ,die Verfahrensmodalitäten, die den Schutz der dem Bürger aus dem Gemeinschaftsrecht erwachsenden Rechte gewährleisten sollen, nach dem Grundsatz der Verfahrensautonomie der Mitgliedsstaaten Sache der innerstaatlichen Rechtsordnung eines jeden Mitgliedsstaats sind“( $)$. Die Verfahrensregelungen, die für die Vollziehung des Unionsrechts gelten, dürfen jedoch nicht ungünstiger sein als diejenigen, die für gleichartige Sachverhalte innerstaatlicher Art gelten (Äquivalenzprinzip). Die nationalen Verfahrensregelungen dürfen die Ausübung der durch die Unionsrechtsordnung verliehenen Rechte nicht praktisch unmöglich machen oder übermäßig erschweren (Effektivitätsprinzip). ${ }^{6}$ )

${ }^{3} \mathrm{Vgl} \mathrm{zB} \S 68$ Abs 3 und 4 Allgemeines Verwaltungsverfahrengesetz (AVG); $§ 530$ Zivilprozessordnung.

${ }^{4}$ EuGH 15.7.1964, 6/64, Costa/E.N.E.L., Slg 1964, 1253 (1270). $57 \mathrm{mwN}$

${ }^{5}$ EuGH 19.9.2006, C-392/04 und C-422/04, i-21 Germany und Arcor, Slg 2006, I-8559 Rz

${ }^{6} \mathrm{Vgl}$ insb EuGH 13.1.2004, C-453/00, Kühne \& Heitz, Slg 2004, I-837; 16.3.2006, C-234/04, Kapferer, Slg 2006, I-2585; 16.11.2010, C-76/10, Pohotovost', Slg 2010, I-11557 Rz 47; vgl auch Kremer, Gemeinschaftsrechtliche Grenzen der Rechtskraft, EuR 2007, 470; Poelzig, Die Aufhebung rechtskräftiger zivilgerichtlicher Urteile unter dem Einfluss des Europäischen Gemeinschaftsrechts, JZ 2007, 858; Griller, „Rechtskraft“ im Gemeinschaftsrecht: Grundlagen und Grenzen in Holoubek/Lang (Hrsg) Rechtskraft im Verwaltungs- und Abgabeverfahren (2008) 55 ff; Schmahl/Köber, Durchbrechung der Rechtskraft nationaler Gerichtsentscheidungen zu Gunsten der Effektivität des Unionsrechts? EuZW 2010, 927. 
Als Zwischenergebnis kann zunächst festgehalten werden, dass die Mitgliedsstaaten auf der einen Seite die Vorrangwirkung des Unionsrechts auf jeder Stufe der Rechtsordnung zu beachten haben, auf der anderen Seite aber ermächtigt sind, das Verfahren der Vollziehung des Unionsrechts nach den Grundsätzen zu regeln, die auch für das nationale Recht gelten. Damit stellt sich die Frage, wie vorzugehen ist, wenn eine verwaltungsbehördliche Entscheidung oder ein gerichtliches Urteil nach nationalem Recht rechtskräftig ist, aber dem Unionsrecht widerspricht. Gilt hier auf Grund der Verfahrensautonomie die nationale Rechtskraftregelung oder - auf Grund des Effektivitätsgrundsatzes die Vorrangwirkung des Unionsrechts? Ein Blick auf die Judikatur des EuGH zeigt, dass der EuGH dieses schwierige Problem von Fall zu Fall zu lösen versucht und im Regelfall einen Mittelweg wählt.

Vordergründig sichtbar wurde diese Problematik erstmals im Fall $\mathrm{Ciola}^{7}$ ). Dieser Fall betraf Österreich. Mit Urteil vom 29. 4. 1999 entschied der EuGH, dass eine verwaltungsbehördliche Entscheidung, die vor dem Beitritt Österreichs zur EU erlassen wurde und nach dem Beitritt Österreichs zur EU gegen die Dienstleistungsfreiheit verstieß, unangewendet zu bleiben hat. Dieser Fall hat zwar in der Literatur großes Aufsehen ausgelöst, dies allerdings zu Unrecht. Man muss nämlich davon ausgehen, dass die Rechtskraft einer verwaltungsbehördlichen Entscheidung nur innerhalb der objektiven und subjektiven Grenzen der Rechtskraft gilt. ${ }^{8}$ ) Zu den objektiven Grenzen gehört die angewendete Rechtslage. Ändert sich diese, so verliert die Rechtskraft eines behördlichen Aktes ihre Wirkung. Genau dies ist im Fall Ciola geschehen: Mit dem Beitritt Österreichs zur EU wurde die Dienstleistungsfreiheit unmittelbar anwendbares Recht, weshalb die Rechtskraft des früher erlassenen Bescheides ihre Wirkungen verlor. Damit stellte sich aber die Frage von unionsrechtlicher Vorrangwirkung und nationaler Rechtskraft nicht mehr. ${ }^{9}$ )

In der Folge kam es freilich zu Verfahren beim EuGH, in denen sich diese Frage sehr wohl stellte; es handelte sich um Fälle in denen eine Entscheidung nationaler Behörden dem Unionsrecht widersprach, dennoch aber nach nationalem Recht rechtskräftig wurde.

\footnotetext{
${ }^{7}$ EuGH 29.4.1999, C-224/97, Ciola, Slg 1999, I-2517.

${ }^{8}$ Vgl dazu Walter/Kolonovits/Muzak/Stöger, Verwaltungsverfahrensrecht ${ }^{9}$ (2011) Rz $480 \mathrm{ff}$.

${ }^{9} \mathrm{Vgl}$ dazu auch Bußjäger, Vorrang des Gemeinschaftsrechts gegenüber individuellen Verwaltungsakten, ecolex 2000, 74 sowie OGH 10 Ob S 172/04y SZ 2005/29.
} 
In ständiger Judikatur betont der EuGH die Bedeutung der Rechtssicherheit, die zu den auch im Unionsrecht anerkannten Grundsätzen gehört. Der EuGH bezieht den Grundsatz der Rechtssicherheit nicht nur auf das nationale Recht sondern ausdrücklich auch auf das Unionsrecht. ${ }^{10}$ ) Aus dem Grundsatz der Rechtssicherheit folge - so der EuGH -, dass das Unionsrecht einem nationalen Recht nicht unter allen Umständen gebiete, von der Anwendung innerstaatlicher Verfahrensvorschriften, auf Grund deren eine Entscheidung Rechtskraft erlangte, abzusehen, selbst wenn dadurch ein Verstoß dieser Entscheidung gegen Unionsrecht abgestellt werden könnte. ${ }^{11}$ )

Bereits im Urteil Kühne \& Heitz hat der $\mathrm{EuGH}^{12}$ ) aber ausgesprochen, dass der Grundsatz der Rechtssicherheit in bestimmten Fällen nur eingeschränkt zum Tragen kommen kann. Der EuGH formulierte vier Voraussetzungen, die kumulativ vorliegen müssen, unter denen rechtskräftige aber unionsrechtswidrige Gerichtsentscheidungen neuerlich überprüft und allenfalls zurückgenommen werden müssen:

$\Rightarrow$ Die Behörde muss nach nationalem Recht befugt sein, eine solche Entscheidung zurückzunehmen;

$\Rightarrow$ die Entscheidung wurde infolge eines Urteils eines in letzter Instanz entscheidenden nationalen Gerichts bestandskräftig;

$\Rightarrow$ das Urteil beruht, wie eine nach seinem Erlass ergangene Entscheidung des EuGH zeigt, auf einer unrichtigen Auslegung des Gemeinschaftsrechts und wurde ohne Ersuchen um Vorabentscheidung gefällt, obwohl die Einholung einer solchen Vorabentscheidung erforderlich gewesen wäre;

$\Rightarrow$ der Betroffene hat sich unmittelbar nachdem er Kenntnis von der besagten Entscheidung des EuGH erlangt hat, an die Verwaltungsbehörde gewandt.

Der EuGH hielt in der Folge an dieser Judikatur fest. ${ }^{13}$ ) Die im Urteil Kühne \& Heitz formulierten Voraussetzungen, die vorliegen müssen, damit ein Staat verpflichtet ist, eine rechtskräftige nationale Gerichtsentscheidung wieder aufzunehmen, prägen die spätere Judikatur.

${ }^{10}$ EuGH 6.10.2009, C-40/08, Asturcom, Slg 2009, I-9579 Rz 35 f mwN; vgl auch OGH 12.6.2012, 4 Ob 83/12b.

${ }^{11}$ EuGH 6.10.2009, C-40/08, Asturcom, Slg 2009, I-9579 Rz 37; ebenso EuGH 19.9.2006, C-392/04 und C-422/04, i-21 Germany und Arcor, Slg 2006, I-8559 Rz 51 sowie EuGH 16.3.2006, C-234/04, Kapferer, Slg 2006, I-2585 Rz 21.

${ }^{12}$ EuGH 13.1.2004, C-453/00, Kühne \& Heitz, Slg 2004, I-837 Rz 28; vgl dazu auch OGH 12.6.2012, 4 Ob 83/12b; VwGH 2003/03/0279 VwSlg 16.992 A; 28.1.2010, 2008/09/0330 sowie Griller in Holoubek/Lang 54 ff; Schmahl/Köber, EuZW 2010, 927 ff.

${ }^{13}$ EuGH 16.3.2006, C-234/04, Kapferer, Slg 2006, I-2585 Rz 23; 19.9.2006, C-392/04 und C-422/04, i-21 Germany und Arcor, Slg 2006, I-8559 Rz 52; vgl auch EuGH 12.2.2008, C-2/06, Kempter, Slg 2008, I-411 Rz 59. 
Die bisherigen Überlegungen haben gezeigt, dass der EuGH den Wert der Rechtssicherheit sehr hoch veranschlagt und demgemäß Eingriffe in die Rechtskraft nationaler gerichtlicher und verwaltungsbehördlicher Entscheidungen nur eingeschränkt für geboten erachtet. Anderes gilt für den Bereich des staatlichen Beihilfenrechts. Im Urteil Lucchini vom 18. 7. 2007 entschied der $\mathrm{EuGH}^{14}$ ), dass ein rechtskräftiges zivilgerichtliches Urteil, mit dem ein Anspruch auf eine staatliche Beihilfe bestätigt wurde, obwohl zuvor durch eine rechtskräftige Entscheidung der Europäischen Kommission deren Unrechtmäßigkeit festgestellt wurde, aufzuheben ist. Eine Bedachtnahme auf die nationale Rechtskraftregelung sei in einem derartigen Fall ein Verstoß gegen Unionsrecht. In einem späteren Urteil qualifizierte es der EuGH als Vertragsverletzung, wenn ein Staat die Rückforderung unionsrechtswidriger Beihilfen nicht effektiv betreibt. Dazu zählt auch, zu verhindern, dass eine nationale gerichtliche Entscheidung in Rechtskraft erwächst. ${ }^{15}$ )

Die deutliche strengere Judikatur des EuGH in Angelegenheiten des Beihilfenrechts ist wohl damit zu erklären, dass die Vollziehung des Beihilfenrechts in die ausschließliche Vollzugskompetenz der Kommission fällt. ${ }^{16}$ ) Deshalb scheint der EuGH dem Grundsatz der Effektivität des Unionsrechts in diesem Bereich größere Bedeutung beizumessen, als dem Grundsatz der Rechtssicherheit. Dazu kommt, dass die betroffenen Mitgliedstaaten in den zit Fällen offenkundig bewusst gegen Unionsrecht verstießen und versuchten, über die Rechtskraft nach nationalem Recht das Unionsrecht zu unterlaufen.

\section{IV}

Sowohl die Effektivität des Unionsrechts wie auch die Wahrung der Rechtssicherheit sind für das Zusammenspiel des Unionsrechts mit den nationalen Rechtsordnungen von fundamentaler Bedeutung. Der EuGH hat sich dieser schwierigen Thematik vorsichtig genähert und bislang $\mathrm{zu}$ ausgewogenen Lösungen gefunden. Dass diese Judikatur nach wie vor von gewissen Unsicherheiten gekennzeichnet ist, darf man dem Gerichtshof nicht vorwerfen. Wenn ausdrückliche Regelungen fehlen und Rechtsfragen unter Bedachtnahme auf allgemeine Rechtsgrundsätze gelöst werden müssen, sind Unsicherheiten die unausweichliche Folge.

\footnotetext{
${ }^{14}$ EuGH 18.7.2007, C-119/05, Lucchini, Slg 2007, I-6199 insb Rz 59 ff.

${ }^{15}$ EuGH 22.12.2010, C-507/08, Kommission/Slowakische Republik, Slg 2010, I-13489 Rz $52 \mathrm{ff}$.

${ }^{16}$ Zutreffend Schmahl/Köber, EuZW 2010, 929.
} 